\title{
Lesions of the Central Nucleus of the Amygdala, but Not the Paraventricular Nucleus of the Hypothalamus, Block the Excitatory Effects of Corticotropin-releasing Factor on the Acoustic Startle Reflex
}

\author{
K. C. Liang, K. R. Melia, S. Campeau, W. A. Falls, M. J. D. Miserendino, and M. Davis \\ Ribicoff Research Facilities of the Connecticut Mental Health Center, Department of Psychiatry, Yale University School of \\ Medicine, New Haven, Connecticut 06508
}

\begin{abstract}
Intracerebroventricular (icv) infusion of corticotropin-releasing factor (CRF) was previously found to produce a longlasting, dose-dependent $(0.1-1.0 \mu \mathrm{g})$ increase in the amplitude of the acoustic startle reflex. The present study sought to determine where in the CNS CRF acts to increase startle. Intracisternal infusion of CRF $(0.1-1.0 \mu \mathrm{g})$ increased startle with a time course and magnitude similar to that produced by icv CRF, unlike intrathecal infusion, which produced a small, more rapid enhancement of startle. While lesions of the paraventricular nucleus of the hypothalamus had no effect on icv CRF-enhanced startle, bilateral lesions of the central nucleus of the amygdala significantly attenuated the excitatory effect of icv CRF but had no effect on intrathecal CRF-enhanced startle. Even though lesions of the amygdala blocked icv CRF-enhanced startle, local infusion of CRF into the amygdala did not significantly elevate startle. The present data indicate that the amygdala is part of the neural circuitry required for icv CRF to elevate startle, but does not appear to be the primary receptor area where CRF acts. The involvement of the amygdala in icv CRF-enhanced startle is consistent with the hypothesis that both the amygdala and CRF are critically involved in fear and stress.
\end{abstract}

We have recently found that intracerebroventricular (icv) infusion of the peptide corticotropin-releasing factor (CRF) produces a dose-dependent increase in the amplitude of the acoustic startle response in rats (Liang et al., 1992). The enhancement appeared approximately 20-30 min after infusion of $1 \mu \mathrm{g} \mathrm{CRF}$, grew steadily to a maximum effect in about $80-90 \mathrm{~min}$, and lasted for at least $6 \mathrm{hr}$. This effect was not dependent on increased sensitization produced by the startle-eliciting stimulus or a blockade of habituation. Peripheral injections of autonomic gan-

Received Sept. 20, 1991; revised Jan. 14, 1992; accepted Jan. 15, 1992.

This research was supported by NIMH Grants MH-25642 and MH-47840, NINCDS Grant NS-18033, Research Scientist Development Award MH-00004, a grant from the Air Force Office of Scientific Research, and the State of Connecticut. W.A.F. and M.J.D.M. were supported by NIMH Training Grant MH 18397. K.C.L. was a visiting scholar sponsored by the National Science Council of the Republic of China. We extend our sincere thanks to Lee Schlesinger, who tested some of the animals used for these studies, and to Leslie Fields for help in typing the manuscript.

Correspondence should be addressed to Dr. Michael Davis, Ribicoff Research Facilities of the Connecticut Mental Health Center, Department of Psychiatry, Yale University School of Medicine, 34 Park Street, New Haven, CT 06508.

Copyright (C) 1992 Society for Neuroscience $0270-6474 / 92 / 122313-08 \$ 05.00 / 0$ glionic blockers attenuated the magnitude of icv CRF-enhanced startle by approximately $35 \%$, suggesting a partial role of the peripheral sympathetic system in the elaboration of icv-CRFenhanced startle. The excitatory effects of CRF could be dosedependently $(25-50 \mu \mathrm{g})$ blocked by icv infusion of the CRF antagonist $\alpha$-helical $\mathrm{CRF}_{9-41}(\alpha \mathrm{hCRF})$, given either $5 \mathrm{~min}$ before or $90 \mathrm{~min}$ after icv CRF, indicating a central site of action. These results confirm and extend those of Swerdlow et al. (1986), who first showed that icv CRF can elevate startle and that this effect can be blocked by icv $\alpha$ hCRF (Swerdlow et al., 1989). The present experiments were designed to begin to evaluate possible CNS sites where icv CRF might act to enhance startle amplitude.

In our test procedure (Liang et al., 1992), CRF-enhanced startle begins to appear about 20-30 min after icv infusion. Because icv drug effects can begin much more rapidly and because the flow of cerebrospinal fluid goes from the lateral ventricle to the fourth ventricle and eventually to the spinal cord, it is possible that the delay in CRF-enhanced startle results from a slow diffusion to more caudal levels of the ventricular system. Hence, the first study tested the effects on startle of CRF given into the fourth ventricle (intracisternal infusion) or the subarachnoid space of the spinal cord (intrathecal infusion).

The paraventricular nucleus of the hypothalamus has a high density of CRF receptors (DeSouza et al., 1985; DeSouza, 1987) and is close to the ventricular surface. In addition to its wellknown role in mediating the release of $\mathrm{ACTH}$ via release of $\mathrm{CRF}$, the paraventricular nucleus projects to a variety of brain areas (cf. Swanson et al., 1983), including the acoustic startle pathway (C. B. Sananes and M. Davis, unpublished observations) and has been implicated in some of the stresslike effects produced by CRF (Krahn et al., 1988). Hence, a second study evaluated whether lesions of the paraventricular nucleus would prevent CRF from elevating startle.

The basolateral nucleus of the amygdala also has relatively high levels of CRF receptors (DeSouza et al., 1985). This amygdaloid nucleus projects heavily to the central nucleus of the amygdala (Krettek and Price, 1978; Otterson, 1982), a structure that repeatedly has been implicated in fear and stress (Gloor, 1960; Kapp et al., 1984, 1990; Sarter and Markowitsch, 1985; Kapp and Pascoe, 1986; Gray, 1989; Davis, 1992). The central nucleus of the amygdala projects directly to the acoustic startle pathway in the brainstem (Rosen et al., 1991), and lesions of the central nucleus of the amygdala, as well as the basolateral nucleus, block the excitatory effects of both conditioned and 
unconditioned fear on startle (Hitchcock and Davis, 1986, 1987; Hitchcock et al., 1989; Sananes and Davis, 1992). Hence, another experiment evaluated whether lesions of the central nucleus of the amygdala would prevent icv CRF-enhanced startle. Because these lesions were found to block CRF-enhanced startle, the effect of direct infusion of CRF into the amygdala on startle was also examined.

\section{Materials and Methods}

\section{Animals}

Male Sprague-Dawley rats (Charles River, Kingston, NY), weighing 280-340 gm, were used as described in the preceding companion article (Liang et al., 1992).

\section{Apparatus}

Startle responses were measured in five identical stabilimeter cages equipped with accelerometers as described in the preceding article (Liang et al., 1992).

\section{Surgery}

Two to three weeks after the rats arrived from the supplier, icv, intracisternal, or intrathecal catheters were implanted. For all implants, rats were anesthetized with chloral hydrate $(400 \mathrm{mg} / \mathrm{kg})$ and positioned in a stereotaxic instrument. Animals were individually housed after surgery and allowed at least 1 week for recovery.

Intracerebroventricular cannula implantation. Methods for design, implantation, and verification of icv cannulas were described in the preceding article (Liang et al., 1992).

Intracisternal cannula implantation. Intracisternal cannulas were composed of $4 \mathrm{~cm}$ and $4 \mathrm{~mm}$ lengths of PE-20 polyethylene tubing, joined by a $4 \mathrm{~mm}$ length of 26 -gauge tubing bent at a right angle. The $4 \mathrm{~mm}$ PE-20 tubing butted against a $3 \times 3 \mathrm{~mm}$ section of rubber band (which served as a gasket). The catheter (secured in a stereotaxic arm parallel to the back of the skull) was inserted through a hole punctured in the atlantooccipital membrane into the cisterna magna until the gasket (which was coated with a thin film of Vaseline) made contact with the back of the skull and the membranes. With the gasket held firmly against the back of the skull (to ensure a tight seal), the catheter was secured with Loctite adhesive to skull screws.

Intrathecal catheter implantation. The catheter used for drug infusion into the lumbar subarachnoid space was composed of an $8.5 \mathrm{~cm}$ length of PE-10 tubing, fused to a $3 \mathrm{~cm}$ length of PE-20 tubing. Methods for catheter construction and implantation have been described previously (Yaksh and Rudy, 1976; Kehne et al., 1986).

Lesions. During the same surgery, rats with icv cannulas were given either bilateral lesions of the central nucleus of the amygdala $(n=14)$ or sham lesions of the amygdala $(n=10)$, or bilateral lesions of the paraventricular nucleus of the hypothalamus $(n=16)$ or sham lesions of the paraventricular nucleus $(n=12)$. For the amygdala lesions, an NE-300 electrode $(0.25 \mathrm{~mm}$ diameter, insulated to within $0.5 \mathrm{~mm}$ of the tip; Rhodes Medical Instrument) was lowered twice into each side of the brain at two different coordinates relative to bregma $(1.4 \mathrm{~mm}$ posterior, $3.5 \mathrm{~mm}$ lateral, $8.5 \mathrm{~mm}$ ventral to the top of the skull; and $3.2 \mathrm{~mm}$ posterior, $4.5 \mathrm{~mm}$ lateral, $7.8 \mathrm{~mm}$ ventral). The lesion current (anode to amygdala, cathode to hindpaw) was $0.1 \mathrm{~mA}, 60-75 \mathrm{sec}$ for each location. Sham lesions followed the same procedure except that no current was passed. For the paraventricular nucleus, cathodal lesions (i.e.. cathode to brain, anode to hindpaw) were used because exploratory studies found anodal lesions of the paraventricular nucleus to be lethal. The coordinates for the paraventricular lesion electrode relative to bregma were $1.7 \mathrm{~mm}$ posterior, $0.5 \mathrm{~mm}$ lateral, and $8.5 \mathrm{~mm}$ ventral. Following these procedures, icv catheters were implanted, as described above. For rats receiving both intrathecal catheters and amygdala ( $n=$ $5)$ or sham amygdala $(n=5)$ lesions, the catheter was implanted 2 weeks after lesion surgery.

Amygdala cannula implantation. Cannulas were made of 23 gauge, thin-wall stainless steel tubing cut into $15 \mathrm{~mm}$ lengths. Each cannula was aimed at the center of the amygdala $(3.0 \mathrm{~mm}$ posterior to bregma, $4.5 \mathrm{~mm}$ lateral, $7.0 \mathrm{~mm}$ ventral to the top of the skull). Cannulas were affixed on the skull with two anchoring screws and dental cement. A $16.5 \mathrm{~mm}$ stylet was inserted into each cannula to maintain patency.

\section{Test procedure and drug administration}

Startle was tested 1 or $2 \mathrm{~d}$ after matching (described in Liang et al., 1992). During testing, rats were placed into the startle cages and, after a 5 min preperiod, were presented with 300 bursts of $105 \mathrm{~dB}, 50 \mathrm{msec}$ white noise. The interstimulus interval was $30 \mathrm{sec}$. Drug infusions occurred immediately after presentation of the 60th stimulus such that 240 stimuli were presented during the $120 \mathrm{~min}$ postinfusion period.

Intracerebroventricular, intracisternal, or intrathecal infusions of CRF [human/rat CRF (Penninsula Laboratory) dissolved in $5 \mu \mathrm{l}$ of artificial cerebrospinal fluid (ACSF)] or ACSF were given remotely without interrupting the progress of stimulus presentation as described in the preceding articlc (Liang et al., 1992).

For intra-amygdala infusions, stimulus presentation paused for approximately $5 \mathrm{~min}$ while rats were taken from the test chamber and infused with $0.5 \mu \mathrm{l}$ of drug or vehicle. A $0.5 \mathrm{~m}$ length of PE-20 tubing connected to a $10 \mu \mathrm{l}$ Hamilton syringe at one end and a 30 gauge dental needle at the other was filled with distilled water, and drug solution was drawn into the tubing from the needle end, separated from the water by a small air bubble. The dental needle was bent such that when inserted into the cannula, the needle tip would protrude beyond the cannula by $1.5 \mathrm{~mm}$. Injections were done manually at a rate of $0.25 \mu \mathrm{l} / \mathrm{min}$. After injection, the needle remained in the cannula for $1 \mathrm{~min}$ before being withdrawn. The stylet was then replaced, the animal was returned to the test chamber, and stimulus presentation resumed immediately.

Verification of cannula placements and histology. Placement of cannulas was verificd by injecting $10 \mu \mathrm{l}$ of methylene bluc dye through the cannulas. Intracerebroventricular cannulas were judged to be properly located if the dye was clearly evident throughout at least one ventricle and the aqueduct of Sylvius. Intracisternal cannulas had to have dye clearly diffused throughout the fourth ventricle, and intrathecal cannulas, throughout the lumbar subarachnoid space both dorsal and ventral to the spinal cord.

Rats with amygdala cannulas or having lesions of the amygdala or paraventricular nucleus were overdosed with chloral hydrate and perfused with $0.9 \%$ saline followed by $10 \%$ formalin. Brains were removed and stored in $30 \%$ sucrose, $10 \%$ formalin solution for at least $48 \mathrm{hr}$. Forty micron brain sections were cut through the cannula tracts or lesion sites, and were stained with cresyl violet.

\section{Data analysis}

Pre- to postchange scores were computed as described in the preceding article (Liang et al., 1992).

\section{Specific procedures}

Effects of intracisternal or intrathecal CRF on startle amplitude (experiment 1). To evaluate the effects on startle amplitude of various doses of CRF given intracisternally, 20 rats with intracisternal cannulas were tested for startle as previously described. They were divided into four groups, each of which received either $5 \mu 1$ of ACSF or $0.1,0.5$, or 1.0 $\mu \mathrm{g}$ of CRF dissolved in $5 \mu \mathrm{l}$ of ACSF ( $n=5$ in each group). Ten other rats with intrathecal catheters were tested for startle after infusion of cither ACSF $(n=5)$ or $1.0 \mu \mathrm{g}$ of CRF $(n=5)$.

Effects of lesions of the hypothalamic paraventricular nucleus or amygdala on icv $C R F$-enhanced startle (experiment 2). Twenty-eight rats were given sham $(n=12)$ or bilateral lesions of the paraventricular nucleus of the hypothalamus $(n=16)$, and 24 rats were given either sham $(n=$ $10)$ or bilateral lesions of the central nucleus of the amygdala $(n=14)$ followed by implantation of icv cannulas in the same surgical session. Two weeks later all animals were given the usual pretest consisting of 60 noise bursts of $105 \mathrm{~dB}$ presented at a $30 \mathrm{sec}$ interstimulus interval; half the animals were then infused with $1.0 \mu \mathrm{g}$ of CRF and half with ACSF, and they were then tested over the next $2 \mathrm{hr}$, as described above. Two to three days later these procedures were repeated except that animals previously infused with CRF were infused with ACSF and vice versa.

Effects of lesions of the amygdala on intrathecal CRF-enhanced startle (experiment 3). Eighteen additional animals received either sham $(n=$ 9) or bilateral amygdala lesions $(n=9)$. Two weeks later, intrathecal catheters were implanted in all animals, as described above. One week after the catheters were implanted, each rat received two startle tests separated by $3-4 \mathrm{~d}$. Five of the nonlesioned rats and five of the amygdala-lesioned rats received intrathecal injections of ACSF in the first test and $1.0 \mu \mathrm{g}$ CRF in the second test, while the remaining rats received 


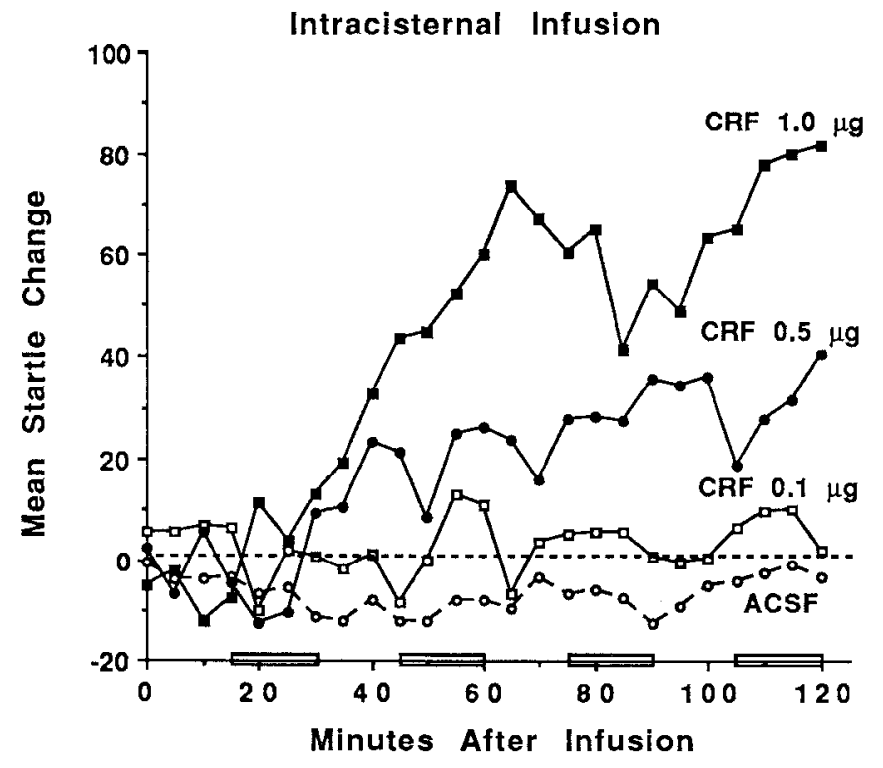

Figure 1. Mean startle change scores of rats receiving intracisternal infusion of ACSF or CRF $(0.1,0.5$, and $1.0 \mu \mathrm{g})$. The rectangular boxes on the $\mathrm{x}$-axis bracket the four time periods sampled for statistical analyses.

injections in the reverse order. Startle testing and injections proceeded as described above.

Effects of local infusion of $C R F$ into the amygdala on startle amplitude (experiment 4). A total of 55 rats received bilateral amygdala cannula implants. Approximately 3 weeks after surgery, rats were tested for acoustic startle as previously described. After presentation of the 60 th stimulus, rats were removed from the chamber and infused bilaterally with $0.01,0.03,0.1$, or $0.3 \mu \mathrm{g}$ of $\mathrm{CRF} / \mathrm{side}$, or vehicle (phosphatebuffered saline), in a volume of $0.5 \mu \mathrm{l} / \mathrm{side}$. The animal was returned to the test cage immediately after injection and presented with the remaining 240 stimuli. Different groups of $7-16$ rats per group were used for each dose or vehicle condition.

\section{Results}

Effects of intracisternal or intrathecal CRF on startle amplitude (experiment 1)

A one-way ANOVA on the preinfusion baseline data revealed no significant differences in the preinfusion mean baseline startle scores of the various groups infused either intracisternally or intrathecally. Therefore, the mean startle change scores will be used for all data presentation and statistical analyses. Figure 1 shows that intracisternal infusion of CRF elevated startle with a time course and dose-response relationship very similar to that previously seen after icv administration. An overall ANOVA on the change scores of the intracisternal data revealed significant effects of CRF dose $[F(3,17)=4.49 ; p<0.02]$, period $[F(3,51)=9.98 ; p<0.001]$, and a dose $\times$ period interaction $[F(9,51)=3.07 ; p<0.005]$. Subsequent post hoc tests revealed significant differences among the various doses during periods $3[F(3,17)=3.11 ; p<0.05]$ and $4[F(3,17)=4.72 ; p<0.01]$.

Figure 2 shows that intrathecal administration of $1 \mu \mathrm{g}$ of CRF also increased acoustic startle amplitude. However, the effect was small relative to that produced by icv or intracisternal administration. In this case there was an overall drug effect $[F(1,16)$ $=12.19 ; p<0.01]$ but no drug $\times$ time interaction $[F(3,48)=$ 1.64], reflecting the fact that the weak excitatory effect of CRF given intrathecally came on more rapidly than that after icv or intracisternal administration.

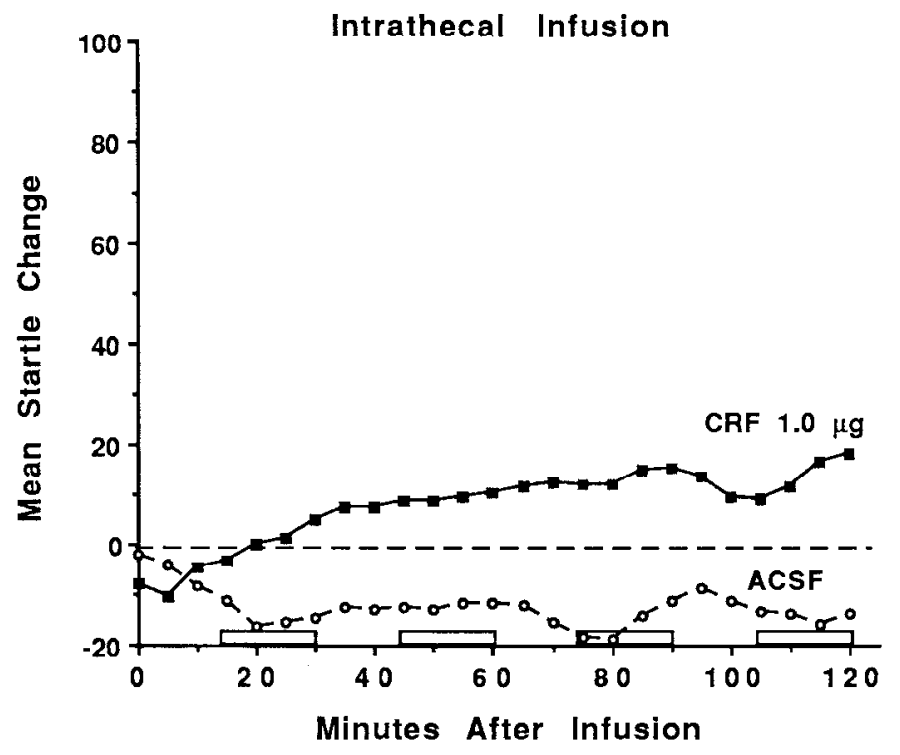

Figure 2. Mean startle change scores of rats receiving intrathecal infusion of ACSF or $1 \mu \mathrm{g}$ CRF.

Effects of lesions of the paraventricular nucleus or amygdala on icv CRF-enhanced startle (experiment 2)

Histology (paraventricular lesions). The cathodal lesions were extremely variable. Ten rats were judged not to have complete lesions of the paraventricular nucleus and were excluded from the analysis. In the remaining six rats, the lesions destroyed all of the paraventricular nucleus bilaterally. In addition, in some animals there was damage to the xiphoid thalamic nucleus, the dorsal and anterior hypothalamic areas, and the most medial aspect of the bed nucleus of the stria terminalis and fornix. In all animals there was significant damage to the dorsal aspect of the periventricular nucleus of the hypothalamus. A photomicrograph from a representative animal is shown in Figure 3.

Bchavior (paraventricular lesions). The preinfusion startle scores (data not shown) of the groups with icv cannulas and either sham lesions or lesions of the paraventricular nucleus of the hypothalamus were analyzed by a $2 \times 2$ (drug $\times$ lesion) ANOVA. Neither the main effects nor the interaction was statistically significant, indicating that lesions of the paraventricular nucleus did not affect baseline startle. The mean postinfusion startle change scores for each group are shown in Figure 4. Consistent with previous studies (Liang et al., 1992), icv CRF caused a time-dependent increase in the amplitude of the acoustic startle reflex relative to infusion of ACSF. Lesions of the paraventricular nucleus of the hypothalamus did not alter either the magnitude or time course of this excitatory effect of CRF.

The postinfusion change scores were analyzed by a $2 \times 2 \times$ 4 (drug $\times$ lesion $\times$ period) repeated-measure ANOVA, with drug (CRF or ACSF) and lesion (sham or lesioned) as betweensubjects variables and period as a within-subjects variable. This revealed a significant effect of drug, reflecting the excitatory effect of CRF on startle $[F(1,16)=19.75 ; p<0.001]$. There was also a significant effect of period $[F(3,48)=3.91 ; p<0.01]$, and drug $\times$ period interaction, reflecting the growth of CRFenhanced startle over the 120 min test session $[F(3,48)=18.21$; $p<0.001]$. Most importantly, there was no overall effect of lesion and no interactions involving lesion (both $F_{\mathrm{s}}<1$ ), reflecting the fact that lesions of the paraventricular nucleus did 


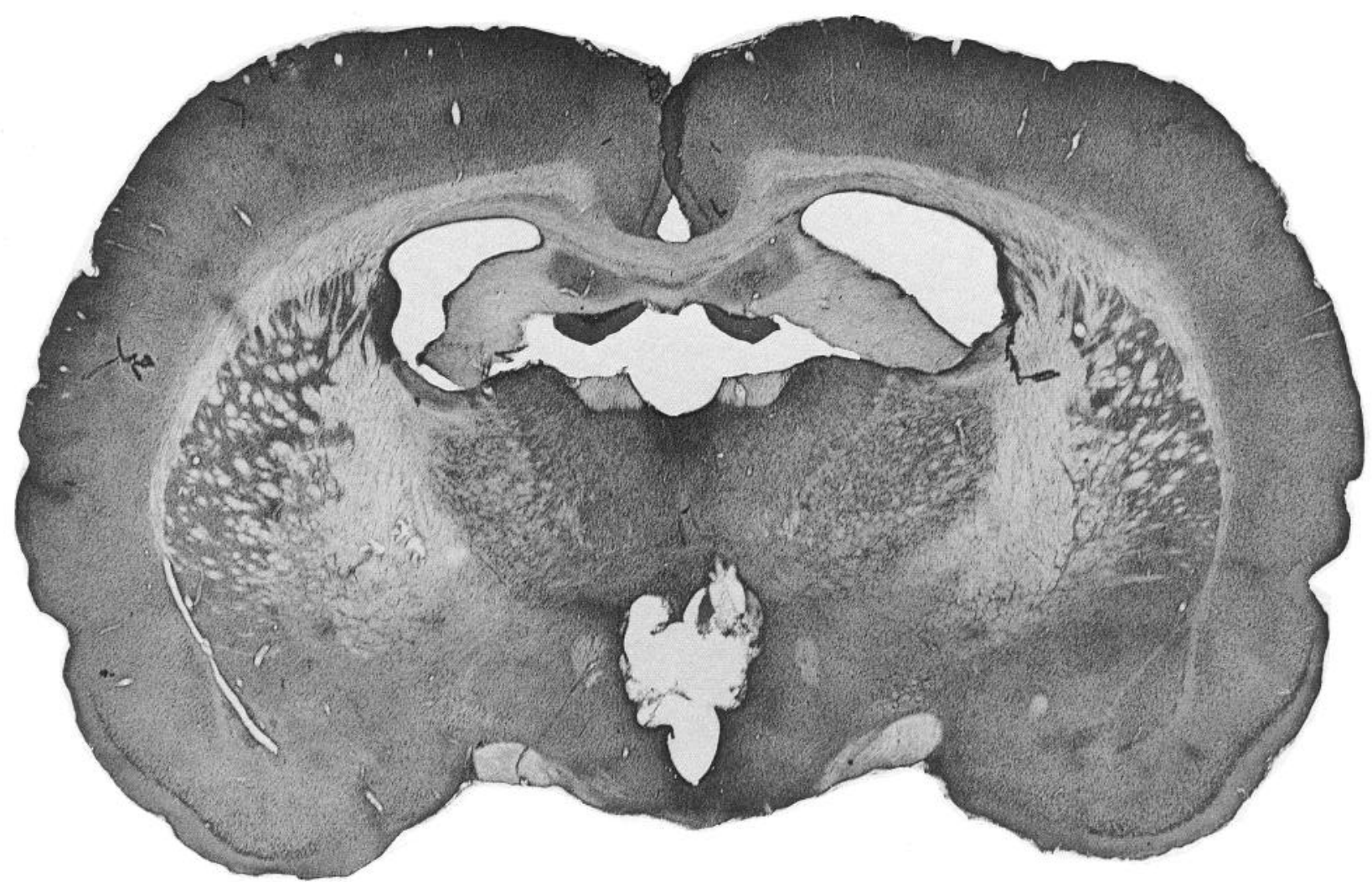

Figure 3. Photomicrograph of an animal judged to have complete, bilateral lesions of the paraventricular nucleus of the hypothalamus.

not alter either the magnitude or time course of CRF-enhanced startle.

Histology (amygdala lesions). Lesions destroyed most of the central nucleus of the amygdala in all the lesioned rats and damaged part of the lateral and basolateral nucleus in most

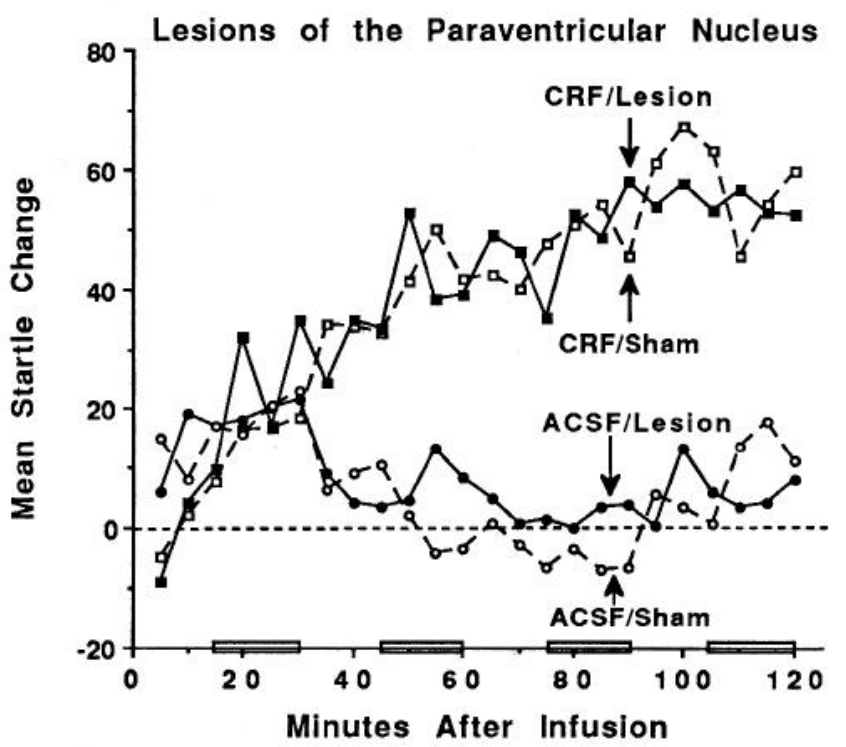

Figure 4. Mean startle change scores following icv infusion of ACSF or $1 \mu \mathrm{g}$ CRF in animals with bilateral lesions of the paraventricular nucleus of the hypothalamus or sham lesions. animals. Lesions from a representative animal are shown in Figure 5.

Behavior (amygdala lesions). The preinfusion scores (data not shown) of the groups with icv cannulas and either amygdala or sham lesions were analyzed by a $2 \times 2$ (drug $\times$ lesion) ANOVA. Neither the main effects nor the interaction effect was statistically significant, indicating that lesions of the amygdala did not alter baseline startle. The mean postinfusion startle change scores for each group are shown in Figure 6. Typical CRF-enhanced startle was found in the sham-operated rats. Amygdala lesions did not affect startle change scores in rats receiving ACSF; however, amygdala lesions nearly eliminated CRF-enhanced startle. The startle change scores of lesioned rats given CRF still were somewhat higher than those of lesioned rats given ACSF, but were much lower than those of the sham-operated rats given CRF.

A $2 \times 2 \times 4$ (drug $\times$ lesion $\times$ period) repeated-measure ANOVA conducted on the postinfusion change scores, with drug (CRF or ACSF) and lesion (sham or lesioned) as between-subjects variables and period as a within-subjects variable, revealed a significant drug $\times$ lesion $\times$ period interaction $[F(3,60)=5.49$; $p<0.002]$. Post hoc analyses indicated that for the shamoperated groups, rats receiving CRF had significantly higher startle change scores than those receiving ACSF at periods 2-4 $[F(1,20)=11.35,31.37,25.42$, respectively; $p<0.005]$. In contrast, in the amygdala-lesioned groups, the difference between CRF and ACSF treatment was not statistically significant at any time period. Further, the sham-operated group given CRF had greater startle change scores than the lesioned group given $\operatorname{CRF}[F(1,20)=13.31 ; p<0.005]$. These findings indicate that 


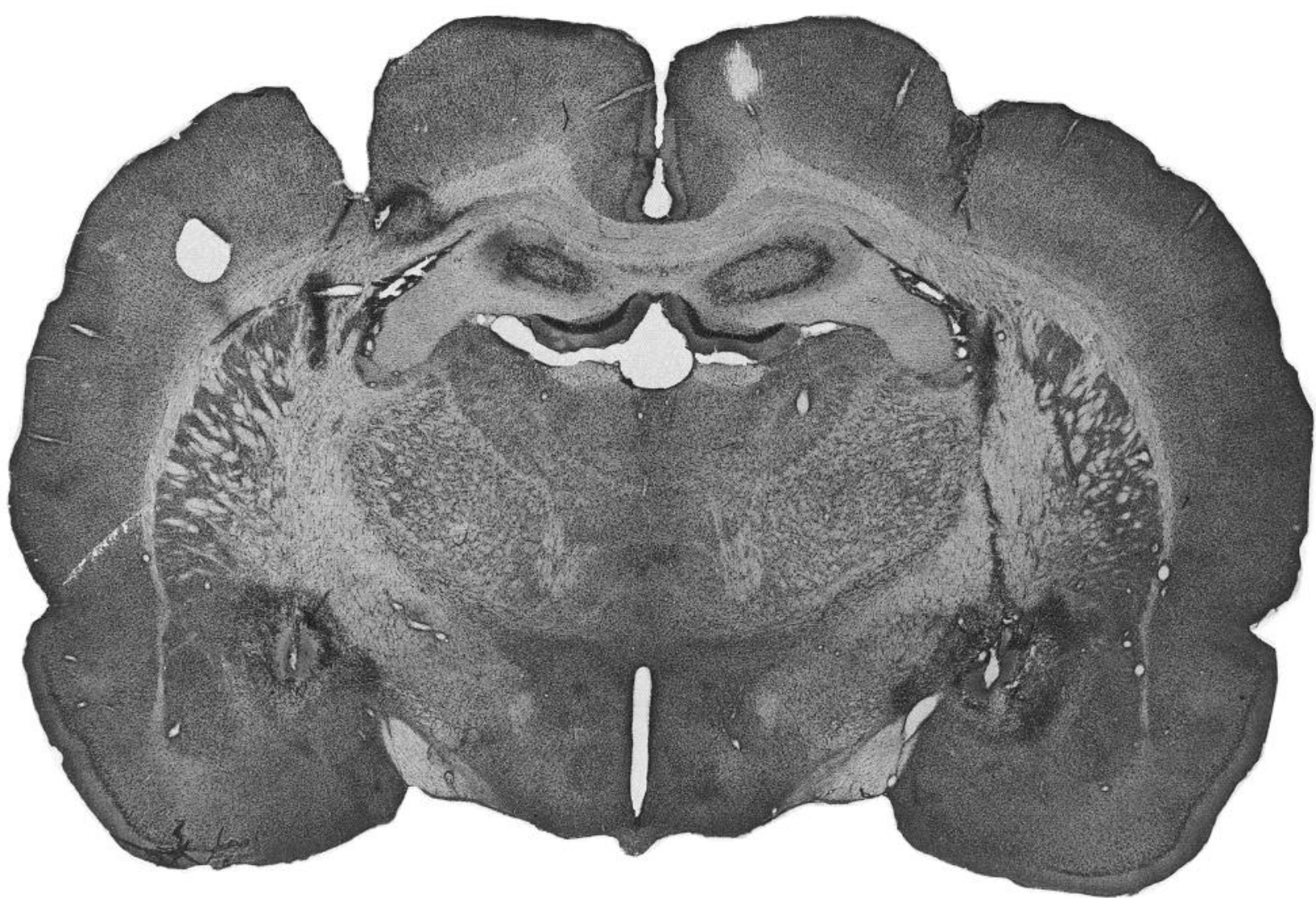

Figure 5. Photomicrograph of an animal judged to have complete, bilateral lesions of the central nucleus of the amygdala.

lesions of the amygdala nearly completely prevented icv CRFenhanced startle.

\section{Effects of lesions of the amygdala on intrathecal $C R F$-enhanced startle (experiment 3 )}

A $2 \times 2$ ANOVA on the preinfusion scores (data not shown) indicated that lesions of the amygdala did not affect baseline startle scores. The mean postinfusion startle change scores are shown in Figure 7. Intrathecal infusion of CRF produced a small but persistent enhancement of startle over the session. Lesions of the amygdala did not affect the startle response of either the ACSF or CRF-infused rats.

A $2 \times 2 \times 4$ (lesion $\times$ drug $\times$ period) repeated-measure ANOVA conducted on the postinfusion change scores, with lesion as a between-subjects variable and drug (ACSF or CRF) and period as within-subjects variables, was performed. Neither the lesion nor the lesion $\times$ drug interaction was significant; however, the significant effect of drug $[F(1,16)=22.07 ; p<$ $0.001]$ indicated that intrathecal CRF enhanced startle. The drug $\times$ period interaction effect was significant $[F(3,48)=7.58 ; p<$ $0.001]$, and a significant linear trend of this interaction effect indicated that the difference between the CRF group and the ACSF group was greater at later periods $[F(1,16)=12.62 ; p<$ 0.005]. Post hoc analyses indicated that intrathecal CRF enhanced startle at all four periods $[F(1,16)=5.57,15.88,31.16$, 22.73 , respectively, for periods $1-4$; all $p$ s $<0.05$ ].

These findings indicate that CRF produced a small but significant enhancement of startle shortly after administration into the lumbar spinal cord, and this enhancement was not altered by amygdala lesions.
Effects of local infusion of CRF into the amygdala on the startle reflex

Histology. The distribution of cannula tips within the amygdala in 33 of the rats that received the CRF is shown in Figure 8 .

Behavior. Mean baseline startle scores (data not shown) of

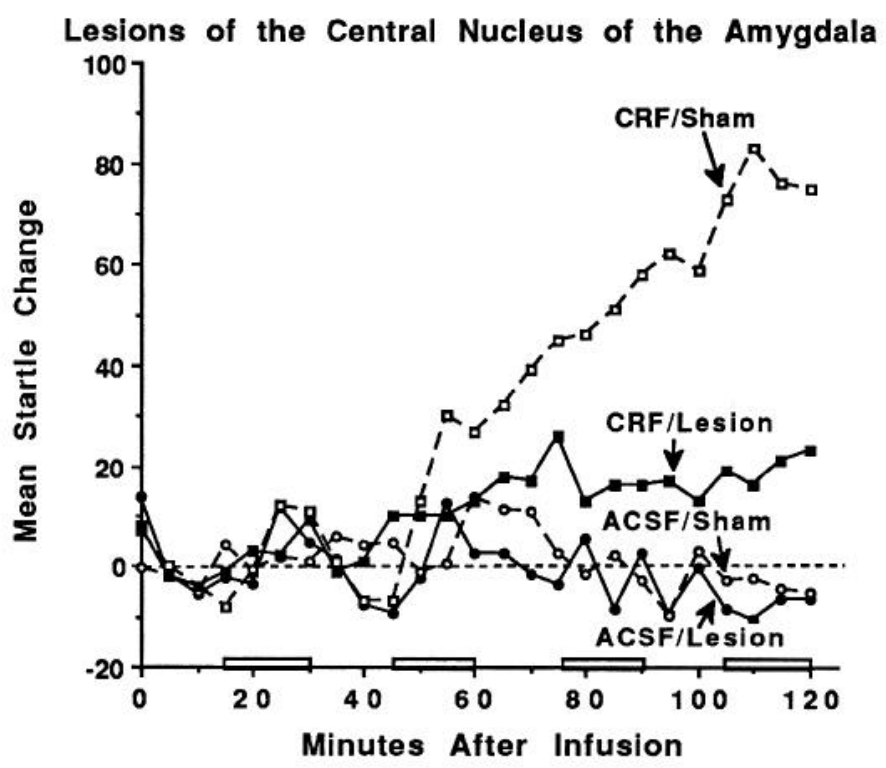

Figure 6. Mean startle change scores following icv infusion of ACSF or $1 \mu \mathrm{g}$ CRF in animals with bilateral lesions of the central nucleus of the amygdala or sham lesions. 


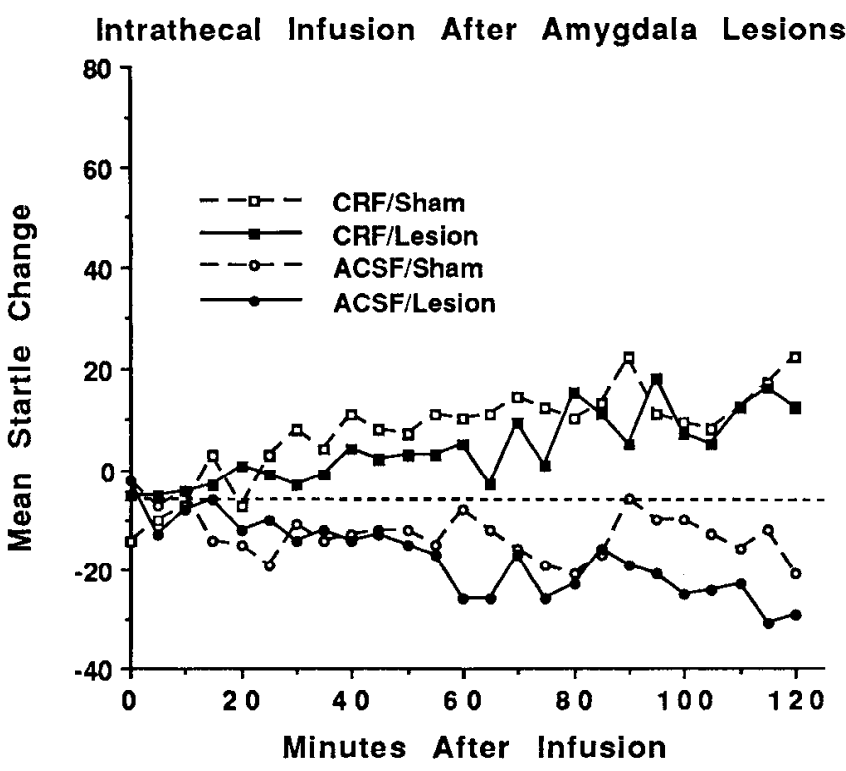

Figure 7. Mean startle change scores following intrathecal infusion of ACSF or $1 \mu \mathrm{g} C R F$ in animals with bilateral lesions of the central nucleus of the amygdala or sham lesions.

various groups were not significantly different. The mean postinfusion startle change scores of the various groups are shown in Figure 9. Intra-amygdala infusions of CRF over a wide range of doses did not produce the pronounced enhancement of startle observed after icv CRF administration. In fact, a decreasing trend in startle over the session was observed in most of the

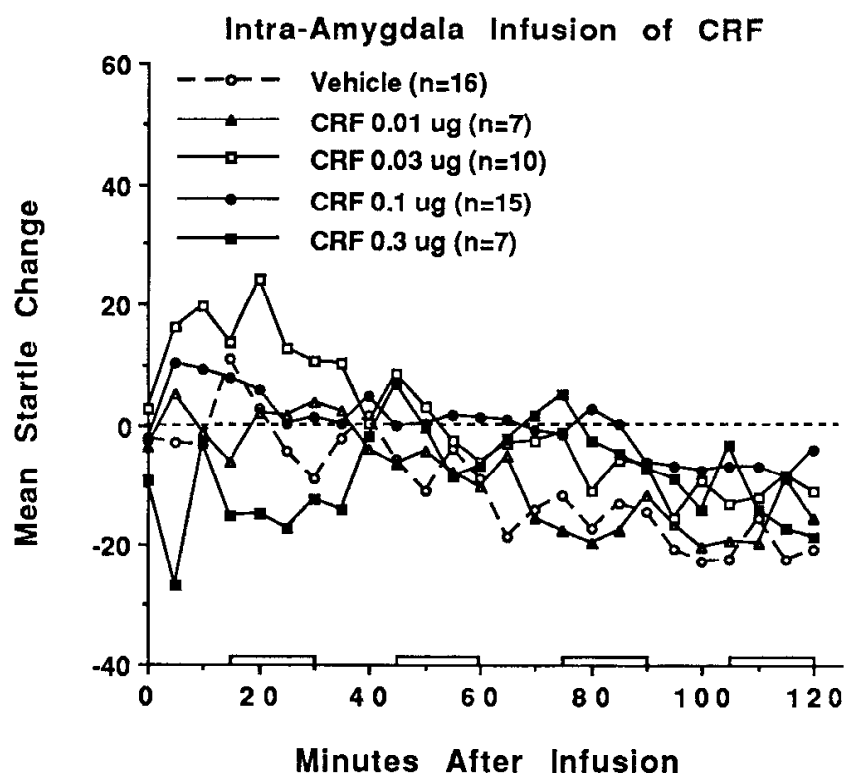

Figure 9. Mean startle change scores following bilateral intra-amygdala infusions of CRF $(0.01,0.03,0.1$, or $0.3 \mu \mathrm{g} /$ side $)$ or ACSF.

groups. Superimposed on this trend, $0.03 \mu \mathrm{g}$ CRF increased, while $0.3 \mu \mathrm{g}$ CRF suppressed, startle at period 1 in comparison with the rats infused with the vehicle for CRF. Rats given 0.1 $\mu \mathrm{g}$ CRF showed some attenuation of the decreasing startle shown in the vehicle-infused rats. Nevertheless, all these differences were rather small relative to that produced by icv CRF in the previous experiments.
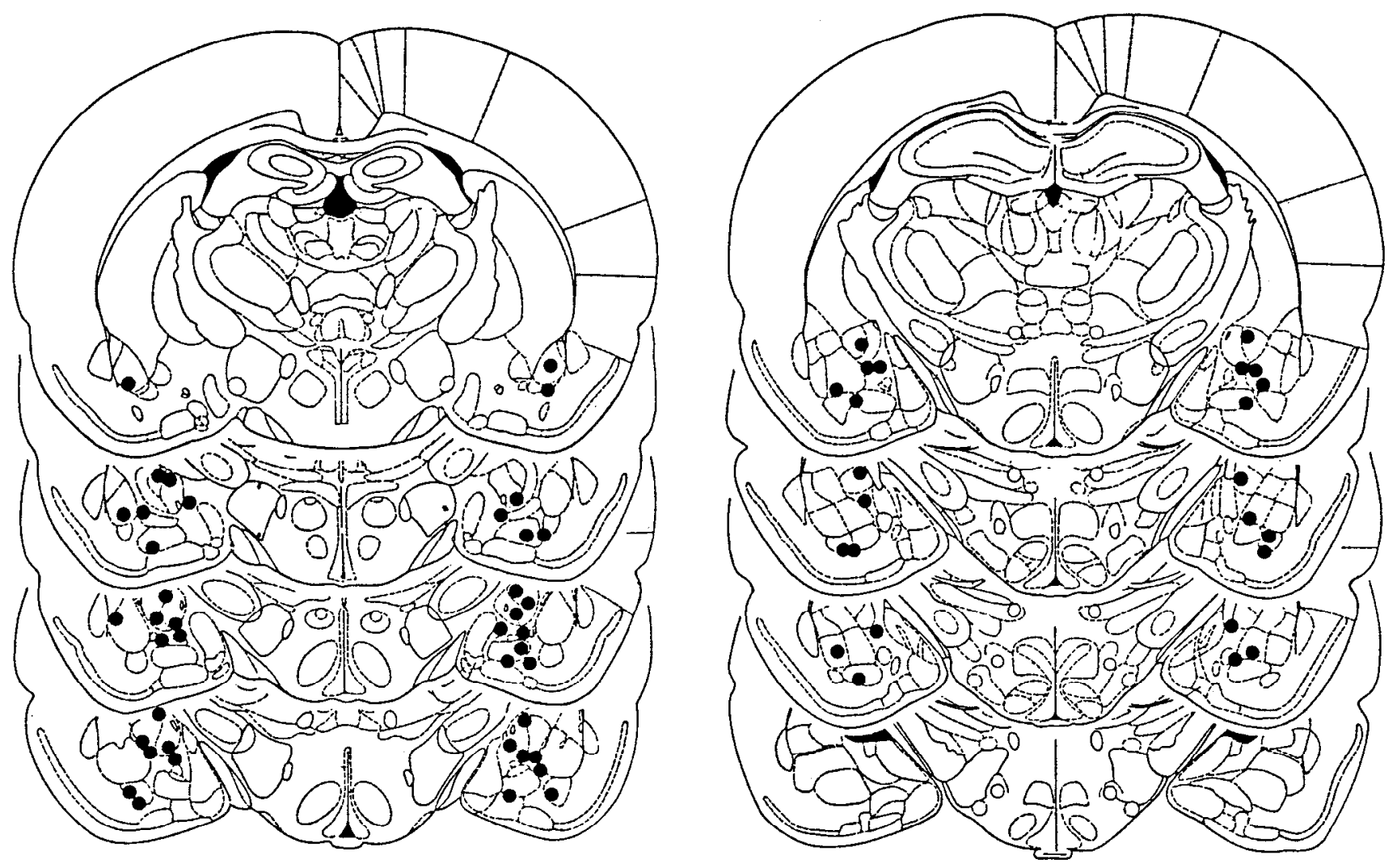

Figure 8. Distribution of cannula tips in the amygdala in the group that received the highest dose of CRF infused into the amygdala. Plates adapted from Paxinos and Watson (1986) with permission from Academic Press. 
The postinfusion change scores were analyzed by a $5 \times 4$ (dose $\times$ period) repeated-measure ANOVA with dose as a between-subjects variable and period as a within-subjects variable. There was a significant effect of period, reflecting the overall decrease in startle responses across the session $[F(3,150)=19.23$; $p<0.001]$. There was no significant effect of dose $[F(4,50)=$ $2.10 ; p<0.10]$; however, the dose $\times$ period interaction was significant $[F(12,150)=3.41 ; p<0.001]$. Post hoc analyses indicated that in comparison with the vehicle-treated group, $0.03 \mu \mathrm{g}$ CRF enhanced startle at period $1[F(1,50)=7.58 ; p<$ $0.01]$ and $0.1 \mu \mathrm{g}$ CRF enhanced startle at periods 3 and $4[F(1,50)$ $=6.77$ and $9.84, p<0.05$ and 0.005 , respectively]. In contrast, the highest dose of CRF $(0.3 \mu \mathrm{g})$ depressed startle at period 1 $[F(1,50)=6.201 ; p<0.05]$ compared to vehicle-treated controls.

The profile and magnitude of these effects do not support the conclusion that the amygdala is the site where the excitatory effect of icv CRF on acoustic startle amplitude is initiated.

\section{Discussion}

Our recent data (Liang et al., 1992) indicated that icv infusion of CRF produced a pronounced, long-lasting enhancement of the acoustic startle reflex. At $1 \mu \mathrm{g}$, CRF-enhanced startle began approximately 20-30 min after infusion and grew steadily to a maximum effect in about $80-90 \mathrm{~min}$. Because icv drug effects can begin more rapidly than $20-30 \mathrm{~min}$ and because the flow of cerebrospinal fluid goes from the lateral ventricle to the fourth ventricle and eventually to the spinal cord, we tested whether the delay in CRF-enhanced startle resulted from diffusion to more caudal levels of the ventricular system. However, the time course and dose-response relationship after intracisternal infusion of CRF were similar to that seen previously after icv CRF. Hence, the delayed onset of icv CRF-enhanced startle cannot be explained by slow diffusion to the fourth ventricle; otherwise, the intracisternal effect would have come on more rapidly. However, it is still possible that CRF diffused rapidly to the fourth ventricle and that the delayed effect resulted from slow diffusion from the fourth ventricle to some other site.

Infusion of CRF into the subarachnoid space of the lumbar spinal cord produced a small enhancement of startle. It is unlikely that this effect was caused by CRF spreading to more rostral parts of the ventricular system, because intrathecal CRFenhanced startle appeared earlier than either the icv or intracisternal effect. Furthermore, lesions of the central nucleus of the amygdala blocked icv but not intrathecal CRF-enhanced startle. CRF receptors are present in the ventral horn (DeSouza et al., 1985) and are involved in depolarization of motor neurons (Bell and DeSouza, 1988). Therefore, CRF injected into the spinal cord could act directly on the spinal motoneurons that mediate the acoustic startle reflex. However, only a small part of icv or intracisternal CRF-enhanced startle could result from diffusion to the subarachnoid space of the spinal cord, because intrathecal infusion of CRF never led to an elevation of startle comparable in magnitude to that after icv or intracisternal infusion.

Previous data indicate the excitatory effects of CRF could be dose-dependently blocked by icv infusion of the CRF antagonist $\alpha$ hCRF given either $5 \mathrm{~min}$ before or $90 \mathrm{~min}$ after icv CRF, suggesting a central site of action. The paraventricular nucleus of the hypothalamus has a high density of CRF receptors (DeSouza et al., 1985; DeSouza, 1987) and is close to the ven- tricular surface. In addition to its well known role in mediating the release of $A C T H$ via release of CRF, the paraventricular nucleus projects to a number of other brain areas (cf. Swanson et al., 1983), including the acoustic startle pathway (Sananes and Davis, unpublished observations), and has been implicated in some of the stresslike effects produced by CRF (Krahn ct al., 1988). However, the present study found that complete lesions of the paraventricular nucleus did not alter either the time course or the magnitude of icv CRF-enhanced startle, ruling out CRF receptors in this nucleus in the initiation of CRF-enhanced startle as well as indicating that this nucleus is not part of the neural circuitry involved in CRF-enhanced startle.

As mentioned earlier, CRF receptors are rich in the amygdala, which is known to play an important modulatory role in the startle reflex. In the present study, bilateral lesions of the central nucleus of the amygdala markedly attenuated icv CRF-enhanced startle, but had no effect on the much smaller enhancement of startle produced by intrathecal CRF. In fact, the magnitude of icv CRF-enhanced startle after amygdala lesions was essentially identical to the magnitude of intrathecal CRF-enhanced startle in sham or amygdala-lesioned animals. This suggests that part of the icv CRF cffect may be attributable to diffusion to the spinal cord, which elevates startle by actions independent of the amygdala, and that the supraspinal effect of icv CRF on startle was completely blocked by lesions of the amygdala. Thus, the amygdala is part of a necessary pathway by which icv CRF enhances the amplitude of the acoustic startle reflex.

It is possible that icv CRF diffuses to and directly stimulates CRF receptors in the amygdala. However, in a series of experiments designed to test this hypothesis, intra-amygdala infusion of a wide range of doses of CRF produced only small, transient effects on startle, and none of these effects were comparable in magnitude or time course to that produced by icv CRF. The lack of a pronounced enhancement of intra-amygdala CRF injection is unlikely to have resulted from insufficient CRF at the critical amygdaloid site, because most of the cannula tips were located in the central and basolateral amygdala nuclei, where CRF receptors are present (DeSouza, 1987). It is conceivable that, because the most posterior parts of the lateral and basolateral nucleus would be closest to the lateral ventricle, more implants would have to be tested at this location before completely ruling out the amygdala as a primary receptor site. However, even the highest dose of CRF $(0.3 \mu \mathrm{g})$ infused into the amygdala did not elevate starlle, but instead produced a significant suppressant effect. These findings suggest that the amygdala, although part of a necessary pathway mediating icv CRFenhanced startle, is not the receptor site where icv CRF initiates this effect.

Thus, it remains to be demonstrated where CRF first acts in the brain to produce enhancement of startle. Based on the present data, we would begin to address this question by locally infusing CRF into brain areas (1) that have high densities of CRF receptors and (2) that project to the amygdala. Local infusion into the relevant area(s) would be expected to (1) elevate startle rapidly after infusion at much lower doses than those required for icv infusion and (2) be blocked by lesions of the amygdala. Preliminary evidence indicates that local infusion of low doses of CRF (1-10 ng) into the vicinity of the parabrachial nucleus or the dorsal lateral tegmental nucleus rapidly (within 2-5 min) elevates startle. We are currently evaluating whether lesions of the amygdala will block these effects and whether 
infusion of the CRF antagonist $\alpha \mathrm{hCRF}$ into these areas will block icv CRF-enhanced startle.

In summary, the present study shows that intracisternal infusion of CRF $(0.1-1.0 \mu \mathrm{g})$ increased startle with a time course and magnitude similar to that previously found for icv CRF. Lesions of the paraventricular nucleus of the hypothalamus had no effect on CRF-enhanced startle, whereas bilateral lesions of the central nucleus of the amygdala nearly climinated the excitatory effect of icv CRF. Intrathecal infusion of $1.0 \mu \mathrm{g}$ of CRF produced a small enhancement of startle that was not altered by amygdala lesions. Even though lesions of the amygdala blocked CRF-enhanced startle, local infusion of CRF into the amygdala did not significantly elevate startle. The involvement of the amygdala in CRF-enhanced startle is consistent with the hypothesis that both the amygdala and CRF are critically involved in fear and stress. The present data indicate that the amygdala is part of the neural circuitry necessary for icv CRF to elevate startle, but does not appear to be the primary receptor area where CRF acts. Because a good deal is known about the neural pathway that mediates the acoustic startle reflex, combined with the fact that this pathway is directly innervated by the amygdala, $\mathrm{CKF}$-enhanced startle may be a useful paradigm for further elucidating the sequence of events that allows icv CRF to produce its profound and long-lasting bchavioral cffects.

\section{References}

Bell JA, DeSouza EB (1988) Functional corticotropin-releasing factor receptors in neonatal rat spinal cord. Peptides 9:1317-1322.

Davis M (1992) The role of the amygdala in conditioned fear. In: The amygdala: neurobiological aspects of emotion, memory and mental dysfunction (Aggleton J, ed), pp 255-305. New York: Wiley.

DeSouza EB (1987) Corticotropin-releasing factor receptors in the rat central nervous system: characterization and regional distribution. J Neurosci 7:88-100.

DeSouza EB, Insel TR, Perrin MH, Rivier J, Vale WW, Kuhar MJ (1985) Corticotropin-releasing factor receptors are widely distributed within the rat central nervous system: an autoradiographic study. $J$ Neurosci 5:3189-3203.

Gloor P (1960) Amygdala. In: Handbook of physiology, Sec I, Neurophysiology (Field J, ed), pp 1395-1420. Washington, DC: American Physiological Society.

Gray TS (1989) Autonomic neuropeptide connections of the amygdala. In: Hans Selye symposia on neuroendocrinology and stress (Tache Y, Morley JE, Brown MR, eds), pp 92-106. New York: Springer.

Hitchcock JM, Davis M (1986) Lesions of the amygdala, but not of the cerebellum or red nucleus, block conditioned fear as measured with the potentiated startle paradigm. Behav Neurosci 100:11-22.

Hitchcock JM, Davis M (1987) Fear-potentiated startle using an auditory conditioned stimulus: effects of lesions of the amygdala. Physiol Behav 39:403-408.
Hitchcock JM, Sananes CB, Davis M (1989) Sensitization of the startle reflex by footshock: blockade by lesions of the central nucleus of the amygdala or its efferent pathway to the brainstem. Behav Neurosci 103:509-518.

Kapp BS, Pascoe JP (1986) Correlation aspects of learning and memorv: vertebrate model systems. In: Learning and memory: a biological view (Martinez JL, Kesner RP, eds), pp 399-440. New York: Academic.

Kapp BS, Pascoe JP, Bixler MA (1984) The amygdala: a neuroanatomical systems approach to its contribution to aversive conditioning. In: The neuropsychology of memory (Butters N, Squire LS, eds), pp 473-488. New York: Guilford.

Kapp BS, Wilson A, Pascoe JP, Supple WF, Whalen PJ (1990) A neuroanatomical systems analysis of conditioned bradycardia in the rabbit. In: Neurocomputation and learning: foundations of adaptive networks (Gabriel M, Moore J, eds), pp 53-90. New York: Bradford.

Kehne JH, Astrachan DI, Astrachan E, Tallman JF, Davis M (1886) The role of spinal cord cyclic AMP in the acoustic startle response in rats. $J$ Neurosci 6:3250-3257.

Krahn DD, Gosnell BA, Levine AS, Morley JE (1988) Behavioral effects of corticotropin-releasing factor: localization and characterization of central effects. Brain Res 443:63-69.

Krettek JE, Price JL (1978) A description of the amygdaloid complex in the rat and cat with observations on intra-amygdaloid axonal connections. J Comp Neurol 178:255-280.

Liang KC, Melia KR, Miserendino M.JD, Falls WA, Campeau S, Davis $M$ (1992) Corticotropin-releasing factor: long-lasting facilitation of the acoustic startle reflex. $J$ Neurosci 12:2303-2312.

Otterson OP (1982) Connections of the amygdala of the rat. IV. Corticoamygdaloid and intraamygdaloid connections as studied with axonal transport of horseradish peroxidase. J Comp Neurol 205:30-48.

Paxinos G, Watson C (1986) The rat brain in stereotaxic coordinates. Orlando, FL: Academic.

Rosen JB, Hitchcock JM, Sananes CB. Miserendino MJD, Davis M (1991) A direct projection from the central nucleus of the amygdala to the acoustic startle pathway: anterograde and retrograde tracing studies. Behav Neurosci 105:817-825.

Sananes CB, Davis M (1992) N-methyl-D-aspartate lesions of the lateral and basolateral nuclei of the amygdala block fear-potentiated startle and shock sensitization of startle. Behav Neurosci 106:72-80.

Sarter M, Markowitsch HJ (1985) Involvement of the amygdala in learning and memory: a critical review, with emphasis on anatomical relations. Behav Neurosci 99:342-380.

Swanson LW, Sawchenko PE, Rivier J, Vale WW (1983) Organization of ovine corticotropin-releasing factor immunoreactive cells and fibers in the rat brain: an immunohistochemical study. Neuroendocrinology 36:165-186.

Swerdlow NR, Geyer MA, Vale WW, Koob GF (1986) Corticotropinreleasing factor potentiates acoustic startle in rats: blockade by chlordiazepoxide. Psychopharmacology 88:147-152.

Swerdlow NR, Britton KT, Koob GF (1989) Potentiation of acoustic startle by corticotropin-releasing factor (CRF) and by fear are both reversed by $\alpha$-helical CRF (9-41). Neuropsychopharmacology 2:285292.

Yaksh TL, Rudy TA (1976) Chronic catheterization of the spinal subarachnoid space. Physiol Behav 17:1031-1036. 